\title{
A Treasure in My Office: Friedel's Publications and Lecture Notes
}

\begin{abstract}
Albert Fert
Received: 10 January 2012 / Accepted: 11 January 2012 / Published online: 5 February 2012

(C) Springer Science+Business Media, LLC 2012
\end{abstract}

I keep an invaluable treasure in my office: a collection of Friedel's papers, not only publications in reviews or in the proceedings of summer schools (les Houches, Enrico Fermi, etc.), but also the transcribed and duplicated notes of his lectures at Orsay for the DEA Physique des Solides. And my most recent use of this treasure? A few months ago, I carefully reread the duplicated notes of Friedel's lecture on the phase shift analysis of scattering in order to work out a theory of the impurity-induced giant Spin Hall Effect, a subject currently in vogue in spintronics. Many theoreticians now use only ab-initio computations of the electronic structure of solids. I was the first to apply Friedel's simple phase shift analysis of electron scattering which the younger generation had forgotten. As this efficient approach, in combination with ab-initio calculations of some parameters, yields better and more transparent results than models using only abinitio calculations, a number of young theoreticians adopted the method and asked me for copies of Friedel's lecture notes. I am pleased to see that a number of publications in my field of research are once again referencing to Friedel's sum rule and his model of phase shift analysis of scattering on impurities. "Monsieur Friedel," (I never said "Jacques" and I am not able to begin doing so now.) would you consider publishing some of your lectures?

What I learned from Friedel's courses and from my discussions with him has played an essential role in the devel- opment of my research. I chose to work in condensed matter physics because Friedel's lectures and André Blandin's advanced topic courses attracted me to this field. (I want to mention that André Blandin, one of Friedel's most talented disciples, had also a very important influence on my own professional formation.) In looking back upon the work in the 1970s of our group of experimentalists at the Laboratoire de Physique des Solides, I clearly see Friedel's beneficial influence on all of us: he gave us a deep knowledge of the fundamentals of solid state physics and a familiarity with solid state theory that was quite unique for experimentalists. It was not only Friedel's lectures that were important to us, but also our many discussions with him at every stage of our research. Friedel advised many researchers during this period, participated in the work of numerous committees, and was certainly very busy, but the door to his office was always open for discussions with us about our recent experimental results. I do not understand how he was able to advise so many researchers. L'Ecole Friedel, Friedel's School, is an appropriate description of the community of physicists whom Friedel influenced over the course of nearly half a century. As I welcome young researchers to search through my collection of Friedel's papers, I am very happy to see how his work continues to influence ongoing developments in Solid State Physics.

\footnotetext{
A. Fert $(\bowtie)$

Universite Paris-Sud 11, Orsay, France

e-mail: albert.fert@thalesgroup.com
} 